\title{
Fixed point iteration processes for asymptotic pointwise nonexpansive mapping in modular function spaces
}

\author{
Buthinah A Bin Dehaish ${ }^{1 *}$ and WM Kozlowski
}

\section{"Correspondence:}

bbindehaish@yahoo.com

${ }^{1}$ Department of Mathematics, King

Abdulaziz University, P.O. Box 53909,

Jeddah 21593, Saudi Arabia

Full list of author information is

available at the end of the article

\section{空 Springer}

\begin{abstract}
Let $L_{\rho}$ be a uniformly convex modular function space with a strong Opial property. Let $T: C \rightarrow C$ be an asymptotic pointwise nonexpansive mapping, where $C$ is a $\rho$-a.e. compact convex subset of $L_{\rho}$. In this paper, we prove that the generalized Mann and Ishikawa processes converge almost everywhere to a fixed point of $T$. In addition, we prove that if $C$ is compact in the strong sense, then both processes converge strongly to a fixed point.

MSC: Primary 47H09; Secondary 47H10

Keywords: fixed point; nonexpansive mapping; fixed point iteration process; Mann process; Ishikawa process; modular function space; Orlicz space; Opial property; uniform convexity
\end{abstract}

\section{Introduction}

In 2008, Kirk and Xu [21] studied the existence of fixed points of asymptotic pointwise nonexpansive mappings $T: C \rightarrow C$, i.e.,

$$
\left\|T^{n}(x)-T^{n}(y)\right\| \leq \alpha_{n}(x)\|x-y\|
$$

where $\lim \sup _{n \rightarrow \infty} \alpha_{n}(x) \leq 1$, for all $x, y \in C$. Their main result (Theorem 3.5) states that every asymptotic pointwise nonexpansive self-mapping of a nonempty, closed, bounded and convex subset $C$ of a uniformly convex Banach space $X$ has a fixed point. As pointed out by Kirk and $\mathrm{Xu}$, asymptotic pointwise mappings seem to be a natural generalization of nonexpansive mappings. The conditions on $\alpha_{n}$ can be for instance expressed in terms of the derivatives of iterations of $T$ for differentiable $T$. In 2009 these results were generalized by Hussain and Khamsi to metric spaces, [9].

In 2011, Khamsi and Kozlowski [18] extended their result proving the existence of fixed points of asymptotic pointwise $\rho$-nonexpansive mappings acting in modular function spaces. The proof of this important theorem is of the existential nature and does not describe any algorithm for constructing a fixed point of an asymptotic pointwise $\rho$ nonexpansive mapping. This paper aims at filling this gap.

Let us recall that modular function spaces are natural generalization of both function and sequence variants of many important, from applications perspective, spaces like Lebesgue, Orlicz, Musielak-Orlicz, Lorentz, Orlicz-Lorentz, Calderon-Lozanovskii spaces 
and many others, see the book by Kozlowski [24] for an extensive list of examples and special cases. There exists an extensive literature on the topic of the fixed point theory in modular function spaces, see, e.g., $[3-5,8,13,14,17-20,24]$ and the papers referenced there.

It is well known that the fixed point construction iteration processes for generalized nonexpansive mappings have been successfully used to develop efficient and powerful numerical methods for solving various nonlinear equations and variational problems, often of great importance for applications in various areas of pure and applied science. There exists an extensive literature on the subject of iterative fixed point construction processes for asymptotically nonexpansive mappings in Hilbert, Banach and metric spaces, see, e.g., $[1,2,6,7,9,12,16,30-36,38-42]$ and the works referred there. Kozlowski proved convergence to fixed point of some iterative algorithms of asymptotic pointwise nonexpansive mappings in Banach spaces [25] and the existence of common fixed points of semigroups of pointwise Lipschitzian mappings in Banach spaces [26]. Recently, weak and strong convergence of such processes to common fixed points of semigroups of mappings in Banach spaces has been demonstrated by Kozlowski and Sims [28].

We would like to emphasize that all convergence theorems proved in this paper define constructive algorithms that can be actually implemented. When dealing with specific applications of these theorems, one should take into consideration how additional properties of the mappings, sets and modulars involved can influence the actual implementation of the algorithms defined in this paper.

The paper is organized as follows:

(a) Section 2 provides necessary preliminary material on modular function spaces.

(b) Section 3 introduces the asymptotic pointwise nonexpansive mappings and related notions.

(c) Section 4 deals with the Demiclosedness Principle which provides a critical stepping stone for proving almost everywhere convergence theorems.

(d) Section 5 utilizes the Demiclosedness Principle to prove the almost everywhere convergence theorem for generalized Mann process.

(e) Section 6 establishes the almost everywhere convergence theorem for generalized Ishikawa process.

(f) Section 7 provides the strong convergence theorem for both generalized Mann and Ishikawa processes for the case of a strongly compact set $C$.

\section{Preliminaries}

Let $\Omega$ be a nonempty set and $\Sigma$ be a nontrivial $\sigma$-algebra of subsets of $\Omega$. Let $\mathcal{P}$ be a $\delta$ ring of subsets of $\Omega$ such that $E \cap A \in \mathcal{P}$ for any $E \in \mathcal{P}$ and $A \in \Sigma$. Let us assume that there exists an increasing sequence of sets $K_{n} \in \mathcal{P}$ such that $\Omega=\bigcup K_{n}$. By $\mathcal{E}$ we denote the linear space of all simple functions with supports from $\mathcal{P}$. By $\mathcal{M}_{\infty}$ we will denote the space of all extended measurable functions, i.e., all functions $f: \Omega \rightarrow[-\infty, \infty]$ such that there exists a sequence $\left\{g_{n}\right\} \subset \mathcal{E},\left|g_{n}\right| \leq|f|$ and $g_{n}(\omega) \rightarrow f(\omega)$ for all $\omega \in \Omega$. By $1_{A}$ we denote the characteristic function of the set $A$.

Definition 2.1 Let $\rho: \mathcal{M}_{\infty} \rightarrow[0, \infty]$ be a nontrivial, convex and even function. We say that $\rho$ is a regular convex function pseudomodular if:

(i) $\rho(0)=0$; 
(ii) $\rho$ is monotone, i.e., $|f(\omega)| \leq|g(\omega)|$ for all $\omega \in \Omega$ implies $\rho(f) \leq \rho(g)$, where $f, g \in \mathcal{M}_{\infty}$;

(iii) $\rho$ is orthogonally subadditive, i.e., $\rho\left(f 1_{A \cup B}\right) \leq \rho\left(f 1_{A}\right)+\rho\left(f 1_{B}\right)$ for any $A, B \in \Sigma$ such that $A \cap B \neq \emptyset, f \in \mathcal{M}_{\infty}$;

(iv) $\rho$ has the Fatou property, i.e., $\left|f_{n}(\omega)\right| \uparrow|f(\omega)|$ for all $\omega \in \Omega$ implies $\rho\left(f_{n}\right) \uparrow \rho(f)$, where $f \in \mathcal{M}_{\infty}$;

(v) $\rho$ is order continuous in $\mathcal{E}$, i.e., $g_{n} \in \mathcal{E}$ and $\left|g_{n}(\omega)\right| \downarrow 0$ implies $\rho\left(g_{n}\right) \downarrow 0$.

Similarly, as in the case of measure spaces, we say that a set $A \in \Sigma$ is $\rho$-null if $\rho\left(g 1_{A}\right)=0$ for every $g \in \mathcal{E}$. We say that a property holds $\rho$-almost everywhere if the exceptional set is $\rho$-null. As usual, we identify any pair of measurable sets whose symmetric difference is $\rho$-null as well as any pair of measurable functions differing only on a $\rho$-null set. With this in mind we define

$$
\mathcal{M}(\Omega, \Sigma, \mathcal{P}, \rho)=\left\{f \in \mathcal{M}_{\infty} ;|f(\omega)|<\infty \rho \text {-a.e. }\right\},
$$

where each $f \in \mathcal{M}(\Omega, \Sigma, \mathcal{P}, \rho)$ is actually an equivalence class of functions equal $\rho$-a.e. rather than an individual function. Where no confusion exists we will write $\mathcal{M}$ instead of $\mathcal{M}(\Omega, \Sigma, \mathcal{P}, \rho)$.

Definition 2.2 Let $\rho$ be a regular function pseudomodular.

(1) We say that $\rho$ is a regular convex function semimodular if $\rho(\alpha f)=0$ for every $\alpha>0$ implies $f=0 \rho$-a.e.;

(2) We say that $\rho$ is a regular convex function modular if $\rho(f)=0$ implies $f=0 \rho$-a.e.;

The class of all nonzero regular convex function modulars defined on $\Omega$ will be denoted by $\Re$.

Let us denote $\rho(f, E)=\rho\left(f 1_{E}\right)$ for $f \in \mathcal{M}, E \in \Sigma$. It is easy to prove that $\rho(f, E)$ is a function pseudomodular in the sense of Def.2.1.1 in [24] (more precisely, it is a function pseudomodular with the Fatou property). Therefore, we can use all results of the standard theory of modular function spaces as per the framework defined by Kozlowski in [22-24].

Remark 2.1 We limit ourselves to convex function modulars in this paper. However, omitting convexity in Definition 2.1 or replacing it by s-convexity would lead to the definition of nonconvex or s-convex regular function pseudomodulars, semimodulars and modulars as in [24].

Definition 2.3 [22-24] Let $\rho$ be a convex function modular.

(a) A modular function space is the vector space $L_{\rho}(\Omega, \Sigma)$, or briefly $L_{\rho}$, defined by

$$
L_{\rho}=\{f \in \mathcal{M} ; \rho(\lambda f) \rightarrow 0 \text { as } \lambda \rightarrow 0\} .
$$

(b) The following formula defines a norm in $L_{\rho}$ (frequently called Luxemurg norm):

$$
\|f\|_{\rho}=\inf \{\alpha>0 ; \rho(f / \alpha) \leq 1\} .
$$

In the following theorem, we recall some of the properties of modular spaces that will be used later on in this paper. 
Theorem 2.1 [22-24] Let $\rho \in \Re$.

(1) $L_{\rho},\|f\|_{\rho}$ is complete and the norm $\|\cdot\|_{\rho}$ is monotone w.r.t. the natural order in $\mathcal{M}$.

(2) $\left\|f_{n}\right\|_{\rho} \rightarrow 0$ if and only if $\rho\left(\alpha f_{n}\right) \rightarrow 0$ for every $\alpha>0$.

(3) If $\rho\left(\alpha f_{n}\right) \rightarrow 0$ for an $\alpha>0$ then there exists a subsequence $\left\{g_{n}\right\}$ of $\left\{f_{n}\right\}$ such that $g_{n} \rightarrow 0 \rho$-a.e.

(4) If $\left\{f_{n}\right\}$ converges uniformly to $f$ on a set $E \in \mathcal{P}$ then $\rho\left(\alpha\left(f_{n}-f\right), E\right) \rightarrow 0$ for every $\alpha>0$.

(5) Let $f_{n} \rightarrow f \rho$-a.e. There exists a nondecreasing sequence of sets $H_{k} \in \mathcal{P}$ such that $H_{k} \uparrow \Omega$ and $\left\{f_{n}\right\}$ converges uniformly to $f$ on every $H_{k}$ (Egoroff theorem).

(6) $\rho(f) \leq \liminf \rho\left(f_{n}\right)$ whenever $f_{n} \rightarrow f \rho$-a.e. (Note: this property is equivalent to the Fatou property.)

(7) Defining $L_{\rho}^{0}=\left\{f \in L_{\rho} ; \rho(f, \cdot)\right.$ is order continuous $\}$ and $E_{\rho}=\left\{f \in L_{\rho} ; \lambda f \in L_{\rho}^{0}\right.$ for every $\left.\lambda>0\right\}$ we have:

(a) $L_{\rho} \supset L_{\rho}^{0} \supset E_{\rho}$,

(b) $E_{\rho}$ has the Lebesgue property, i.e., $\rho\left(\alpha f, D_{k}\right) \rightarrow 0$ for $\alpha>0, f \in E_{\rho}$ and $D_{k} \downarrow \emptyset$.

(c) $E_{\rho}$ is the closure of $\mathcal{E}$ (in the sense of $\|\cdot\|_{\rho}$ ).

The following definition plays an important role in the theory of modular function spaces.

Definition 2.4 Let $\rho \in \Re$. We say that $\rho$ has the $\Delta_{2}$-property if

$$
\sup _{n} \rho\left(2 f_{n}, D_{k}\right) \rightarrow 0
$$

whenever $D_{k} \downarrow \emptyset$ and $\sup _{n} \rho\left(f_{n}, D_{k}\right) \rightarrow 0$.

Theorem 2.2 Let $\rho \in \Re$. The following conditions are equivalent:

(a) $\rho$ has $\Delta_{2}$,

(b) $L_{\rho}^{0}$ is a linear subspace of $L_{\rho}$,

(c) $L_{\rho}=L_{\rho}^{0}=E_{\rho}$,

(d) if $\rho\left(f_{n}\right) \rightarrow 0$, then $\rho\left(2 f_{n}\right) \rightarrow 0$,

(e) if $\rho\left(\alpha f_{n}\right) \rightarrow 0$ for an $\alpha>0$, then $\left\|f_{n}\right\|_{\rho} \rightarrow 0$, i.e., the modular convergence is equivalent to the norm convergence.

We will also use another type of convergence which is situated between norm and modular convergence. It is defined, among other important terms, in the following definition.

Definition 2.5 Let $\rho \in \Re$.

(a) We say that $\left\{f_{n}\right\}$ is $\rho$-convergent to $f$ and write $f_{n} \rightarrow f(\rho)$ if and only if $\rho\left(f_{n}-f\right) \rightarrow 0$.

(b) A sequence $\left\{f_{n}\right\}$ where $f_{n} \in L_{\rho}$ is called $\rho$-Cauchy if $\rho\left(f_{n}-f_{m}\right) \rightarrow 0$ as $n, m \rightarrow \infty$.

(c) A set $B \subset L_{\rho}$ is called $\rho$-closed if for any sequence of $f_{n} \in B$, the convergence $f_{n} \rightarrow f(\rho)$ implies that $f$ belongs to $B$.

(d) A set $B \subset L_{\rho}$ is called $\rho$-bounded if $\sup \{\rho(f-g) ; f \in B, g \in B\}<\infty$.

(e) A set $B \subset L_{\rho}$ is called strongly $\rho$-bounded if there exists $\beta>1$ such that $M_{\beta}(B)=\sup \{\rho(\beta(f-g)) ; f \in B, g \in B\}<\infty$.

(f) A set $B \subset L_{\rho}$ is called $\rho$-compact if for any $\left\{f_{n}\right\}$ in $C$ there exists a subsequence $\left\{f_{n_{k}}\right\}$ and an $f \in C$ such that $\rho\left(f_{n_{k}}-f\right) \rightarrow 0$. 
(g) A set $C \subset L_{\rho}$ is called $\rho$-a.e. closed if for any $\left\{f_{n}\right\}$ in $C$ which $\rho$-a.e. converges to some $f$, then we must have $f \in C$.

(h) A set $C \subset L_{\rho}$ is called $\rho$-a.e. compact if for any $\left\{f_{n}\right\}$ in $C$, there exists a subsequence $\left\{f_{n_{k}}\right\}$ which $\rho$-a.e. converges to some $f \in C$.

(i) Let $f \in L_{\rho}$ and $C \subset L_{\rho}$. The $\rho$-distance between $f$ and $C$ is defined as

$$
d_{\rho}(f, C)=\inf \{\rho(f-g) ; g \in C\} .
$$

Let us note that $\rho$-convergence does not necessarily imply $\rho$-Cauchy condition. Also, $f_{n} \rightarrow f$ does not imply in general $\lambda f_{n} \rightarrow \lambda f, \lambda>1$. Using Theorem 2.1 , it is not difficult to prove the following:

\section{Proposition 2.1 Let $\rho \in \Re$.}

(i) $L_{\rho}$ is $\rho$-complete,

(ii) $\rho$-balls $B_{\rho}(x, r)=\left\{y \in L_{\rho} ; \rho(x-y) \leq r\right\}$ are $\rho$-closed and $\rho$-a.e. closed.

Let us compare different types of compactness introduced in Definition 2.5.

Proposition 2.2 Let $\rho \in \Re$. The following relationships hold for sets $C \subset L_{\rho}$ :

(i) If $C$ is $\rho$-compact, then $C$ is $\rho$-a.e. compact.

(ii) If $C$ is $\|\cdot\|_{\rho}$-compact, then $C$ is $\rho$-compact.

(iii) If $\rho$ satisfies $\Delta_{2}$, then $\|\cdot\|_{\rho}$-compactness and $\rho$-compactness are equivalent in $L_{\rho}$.

Proof

(i) follows from Theorem 2.1 part (3).

(ii) follows from Theorem 2.1 part (2).

(iii) follows from (2.2) and from Theorem 2.2 part (e).

\section{Asymptotic pointwise nonexpansive mappings}

Let us recall the modular definitions of asymptotic pointwise nonexpansive mappings and associated notions, [18].

Definition 3.1 Let $\rho \in \Re$ and let $C \subset L_{\rho}$ be nonempty and $\rho$-closed. A mapping $T: C \rightarrow$ $C$ is called an asymptotic pointwise mapping if there exists a sequence of mappings $\alpha_{n}$ : $C \rightarrow[0, \infty)$ such that

$$
\rho\left(T^{n}(f)-T^{n}(g)\right) \leq \alpha_{n}(f) \rho(f-g) \quad \text { for any } f, g \in L_{\rho} .
$$

(i) If $\alpha_{n}(f)=1$ for every $f \in L_{\rho}$ and every $n \in \mathbb{N}$, then $T$ is called $\rho$-nonexpansive or shortly nonexpansive.

(ii) If $\left\{\alpha_{n}\right\}$ converges pointwise to $\alpha: C \rightarrow[0,1)$, then $T$ is called asymptotic pointwise contraction.

(iii) If $\lim \sup _{n \rightarrow \infty} \alpha_{n}(f) \leq 1$ for any $f \in L_{\rho}$, then $T$ is called asymptotic pointwise nonexpansive.

(iv) If $\lim \sup _{n \rightarrow \infty} \alpha_{n}(f) \leq k$ for any $f \in L_{\rho}$ with $0<k<1$, then $T$ is called strongly asymptotic pointwise contraction. 
Denoting $a_{n}(x)=\max \left(\alpha_{n}(x), 1\right)$, we note that without loss of generality we can assume that $T$ is asymptotically pointwise nonexpansive if

$$
\begin{aligned}
& \rho\left(T^{n}(f)-T^{n}(g)\right) \leq a_{n}(f) \rho(f-g) \quad \text { for all } f, g \in C, n \in \mathbb{N}, \\
& \lim _{n \rightarrow \infty} a_{n}(f)=1, a_{n}(f) \geq 1 \quad \text { for all } f \in C, \text { and } n \in \mathbb{N} .
\end{aligned}
$$

Define $b_{n}(f)=a_{n}(f)-1$. In view of (3.2), we have

$$
\lim _{n \rightarrow \infty} b_{n}(f)=0 .
$$

The above notation will be consistently used throughout this paper.

By $\mathcal{T}(C)$ we will denote the class of all asymptotic pointwise nonexpansive mappings $T: C \rightarrow C$.

In this paper, we will impose some restrictions on the behavior of $a_{n}$ and $b_{n}$. This type of assumptions is typical for controlling the convergence of iterative processes for asymptotically nonexpansive mappings, see, e.g., [25].

Definition 3.2 Define $\mathcal{T}_{r}(C)$ as a class of all $T \in \mathcal{T}(C)$ such that

$$
\begin{aligned}
& \sum_{n=1}^{\infty} b_{n}(x)<\infty \text { for every } x \in C, \\
& a_{n} \text { is a bounded function for every } n \geq 1 .
\end{aligned}
$$

We recall the following concepts related to the modular uniform convexity introduced in [18]:

Definition 3.3 Let $\rho \in \Re$. We define the following uniform convexity type properties of the function modular $\rho$ : Let $t \in(0,1), r>0, \varepsilon>0$. Define

$$
D_{1}(r, \varepsilon)=\left\{(f, g) ; f, g \in L_{\rho}, \rho(f) \leq r, \rho(g) \leq r, \rho(f-g) \geq \varepsilon r\right\} .
$$

Let

$$
\delta_{1}^{t}(r, \varepsilon)=\inf \left\{1-\frac{1}{r} \rho(t f+(1-t) g) ;(f, g) \in D_{1}(r, \varepsilon)\right\}, \quad \text { if } D_{1}(r, \varepsilon) \neq \emptyset,
$$

and $\delta_{1}(r, \varepsilon)=1$ if $D_{1}(r, \varepsilon)=\emptyset$. We will use the following notational convention: $\delta_{1}=\delta_{1}^{\frac{1}{2}}$.

Definition 3.4 We say that $\rho$ satisfies (UC1) if for every $r>0, \varepsilon>0, \delta_{1}(r, \varepsilon)>0$. Note that for every $r>0, D_{1}(r, \varepsilon) \neq \emptyset$, for $\varepsilon>0$ small enough. We say that $\rho$ satisfies (UUC1) if for every $s \geq 0, \varepsilon>0$ there exists $\eta_{1}(s, \varepsilon)>0$ depending only on $s$ and $\varepsilon$ such that

$$
\delta_{1}(r, \varepsilon)>\eta_{1}(s, \varepsilon)>0 \quad \text { for any } r>s .
$$

We will need the following result whose proof is elementary. Note that for $t=\frac{1}{2}$, this result follows directly from Definition 3.4. 
Lemma 3.1 Let $\rho \in \Re$ be (UUC1) and let $t \in(0,1)$. Then for every $s>0, \varepsilon>0$ there exists $\eta_{1}^{t}(s, \varepsilon)>0$ depending only on $s$ and $\varepsilon$ such that

$$
\delta_{1}^{t}(r, \varepsilon)>\eta_{1}^{t}(s, \varepsilon)>0 \quad \text { for any } r>s .
$$

The notion of bounded away sequences of real numbers will be used extensively throughout this paper.

Definition 3.5 A sequence $\left\{t_{n}\right\} \subset(0,1)$ is called bounded away from 0 if there exists $0<$ $a<1$ such that $t_{n} \geq a$ for every $n \in \mathbb{N}$. Similarly, $\left\{t_{n}\right\} \subset(0,1)$ is called bounded away from 1 if there exists $0<b<1$ such that $t_{n} \leq b$ for every $n \in \mathbb{N}$.

We will need the following generalization of Lemma 4.1 from [18] and being a modular equivalent of a norm property in uniformly convex Banach spaces, see, e.g., [36].

Lemma 3.2 Let $\rho \in \Re$ be $(U U C 1)$ and let $\left\{t_{k}\right\} \subset(0,1)$ be bounded away from 0 and 1 . If there exists $R>0$ such that

$$
\begin{aligned}
& \limsup _{n \rightarrow \infty} \rho\left(f_{n}\right) \leq R, \quad \limsup _{n \rightarrow \infty} \rho\left(g_{n}\right) \leq R, \\
& \lim _{n \rightarrow \infty} \rho\left(t_{n} f_{n}+\left(1-t_{n}\right) g_{n}\right)=R,
\end{aligned}
$$

then

$$
\lim _{n \rightarrow \infty} \rho\left(f_{n}-g_{n}\right)=0
$$

Proof Assume to the contrary that this is not the case and fix an arbitrary $\gamma>0$. Passing to a subsequence if necessary, we may assume that there exists an $\varepsilon>0$ such that

$$
\rho\left(f_{n}\right) \leq R+\gamma, \quad \rho\left(g_{n}\right) \leq R+\gamma,
$$

while

$$
\rho\left(f_{n}-g_{n}\right) \geq(R+\gamma) \varepsilon
$$

Since $\left\{t_{n}\right\}$ is bounded away from 0 and 1 there exist $0<a<b<1$ such that $a \leq t_{n} \leq b$ for all natural $n$. Passing to a subsequence if necessary, we can assume that $t_{n} \rightarrow t_{0} \in[a, b]$. For every $t \in[0,1]$ and $f, g \in D_{1}(R+\gamma, \varepsilon)$, let us define $\lambda_{f, g}(t)=\rho(t f+(1-t) g)$. Observe that the function $\lambda_{f, g}:[0,1] \rightarrow[0, R+\gamma]$ is a convex function. Hence that the function

$$
\lambda(t)=\sup \left\{\lambda_{f, g}(t): f, g \in D_{1}(R+\gamma, \varepsilon)\right\}
$$

is also convex on $[0,1]$, and consequently, it is a continuous function on $[a, b]$. Noting that

$$
\delta_{1}^{t}(R+\gamma, \varepsilon)=1-\frac{1}{r} \lambda(t)
$$


we conclude that $\delta_{1}^{t}(R+\gamma, \varepsilon)$ is a continuous function of $t \in[a, b]$. Hence

$$
\lim _{n \rightarrow \infty} \delta_{1}^{t_{n}}(R+\gamma, \varepsilon)=\delta_{1}^{t_{0}}(R+\gamma, \varepsilon) .
$$

By (3.8) and (3.9)

$$
\delta_{1}^{t_{n}}(R+\gamma, \varepsilon) \leq 1-\frac{1}{R+\gamma} \rho\left(t_{n} f_{n}+\left(1-t_{n}\right) g_{n}\right) .
$$

By (3.12) the left-hand side of (3.13) tends to $\delta_{1}^{t_{0}}(R+\gamma, \varepsilon)$ as $n \rightarrow \infty$ while the right-hand side tends to $\frac{\gamma}{R+\gamma}$ in view of (3.7). Hence

$$
\delta_{1}^{t_{0}}(R+\gamma, \varepsilon) \leq \frac{\gamma}{R+\gamma} .
$$

By $(U U C 1)$ and by Lemma 3.1, there exists $\eta_{1}^{t_{0}}(R, \varepsilon)>0$ satisfying

$$
0<\eta_{1}^{t_{0}}(R, \varepsilon) \leq \delta_{1}^{t_{0}}(R+\gamma, \varepsilon) .
$$

Combining (3.14) with (3.15) we get

$$
0<\eta_{1}^{t_{0}}(R, \varepsilon) \leq \frac{\gamma}{R+\gamma}
$$

Letting $\gamma \rightarrow 0$ we get a contradiction which completes the proof.

Let us introduce a notion of a $\rho$-type, a powerful technical tool which will be used in the proofs of our fixed point results.

Definition 3.6 Let $K \subset L_{\rho}$ be convex and $\rho$-bounded. A function $\tau: K \rightarrow[0, \infty]$ is called a $\rho$-type (or shortly a type) if there exists a sequence $\left\{y_{n}\right\}$ of elements of $K$ such that for any $z \in K$ there holds

$$
\tau(z)=\limsup _{n \rightarrow \infty} \rho\left(y_{n}-z\right) .
$$

Note that $\tau$ is convex provided $\rho$ is convex. A typical method of proof for the fixed point theorems in Banach and metric spaces is to construct a fixed point by finding an element on which a specific type function attains its minimum. To be able to proceed with this method, one has to know that such an element indeed exists. This will be the subject of Lemma 3.3 below. First, let us recall the definition of the Opial property and the strong Opial property in modular function spaces, $[15,17]$.

Definition 3.7 We say that $L_{\rho}$ satisfies the $\rho$-a.e. Opial property if for every $\left\{f_{n}\right\} \in L_{\rho}$ which is $\rho$-a.e. convergent to 0 such that there exists a $\beta>1$ for which

$$
\sup _{n}\left\{\rho\left(\beta f_{n}\right)\right\}<\infty,
$$

the following inequality holds for any $g \in E_{\rho}$ not equal to 0

$$
\liminf _{n \rightarrow \infty} \rho\left(f_{n}\right) \leq \liminf _{n \rightarrow \infty} \rho\left(f_{n}+g\right) .
$$


Definition 3.8 We say that $L_{\rho}$ satisfies the $\rho$-a.e. strong Opial property if for every $\left\{f_{n}\right\} \in$ $L_{\rho}$ which is $\rho$-a.e. convergent to 0 such that there exists a $\beta>1$ for which

$$
\sup _{n}\left\{\rho\left(\beta f_{n}\right)\right\}<\infty
$$

the following equality holds for any $g \in E_{\rho}$

$$
\liminf _{n \rightarrow \infty} \rho\left(f_{n}+g\right)=\liminf _{n \rightarrow \infty} \rho\left(f_{n}\right)+\rho(g)
$$

Remark 3.1 Note that the $\rho$-a.e. Strong Opial property implies $\rho$-a.e. Opial property [15].

Remark 3.2 Also, note that, by virtue of Theorem 2.1 in [15], every convex, orthogonally additive function modular $\rho$ has the $\rho$-a.e. strong Opial property. Let us recall that $\rho$ is called orthogonally additive if $\rho(f, A \cup B)=\rho(f, A)+\rho(f, B)$ whenever $A \cap B=\emptyset$. Therefore, all Orlicz and Musielak-Orlicz spaces must have the strong Opial property.

Note that the Opial property in the norm sense does not necessarily hold for several classical Banach function spaces. For instance, the norm Opial property does not hold for $L^{p}$ spaces for $1 \leq p \neq 2$ while the modular strong Opial property holds in $L^{p}$ for all $p \geq 1$.

Lemma 3.3 [27] Let $\rho \in \Re$. Assume that $L_{\rho}$ has the $\rho$-a.e. strong Opial property. Let $C \subset$ $E_{\rho}$ be a nonempty, strongly $\rho$-bounded and $\rho$-a.e. compact convex set. Then any $\rho$-type defined in $C$ attains its minimum in $C$.

Let us finish this section with the fundamental fixed point existence theorem which will be used in many places in the current paper.

Theorem 3.1 [18] Assume $\rho \in \mathfrak{R}$ is (UUC1). Let $C$ be a $\rho$-closed $\rho$-bounded convex nonempty subset. Then any $T: C \rightarrow C$ asymptotically pointwise nonexpansive has a fixed point. Moreover, the set of all fixed points $\operatorname{Fix}(T)$ is $\rho$-closed.

\section{Demiclosedness Principle}

The following modular version of the Demiclosedness Principle will be used in the proof of our convergence Theorem 5.1. Our proof the Demiclosedness Principle uses the parallelogram inequality valid in the modular spaces with the (UUC1) property (see Lemma 4.2 in [18]). We start with a technical result which will be used in the proof of Theorem 4.1.

Lemma 4.1 Let $\rho \in \Re$. Let $C \subset L_{\rho}$ be a convex set, and let $T \in \mathcal{T}_{r}(C)$. If $\left\{x_{k}\right\}$ is a $\rho$ approximate fixed point sequence for $T$, that is, $\rho\left(T\left(x_{k}\right)-x_{k}\right) \rightarrow 0$ as $k \rightarrow \infty$, then for every fixed $m \in \mathbb{N}$ there holds

$$
\rho\left(\frac{T^{m}\left(x_{k}\right)-x_{k}}{m}\right) \rightarrow 0,
$$

as $k \rightarrow \infty$. 
Proof It follows from 3.5 that there exists a finite constant $M>0$ such that

$$
\sum_{j=1}^{m-1} \sup \left\{a_{j}(x) ; x \in C\right\} \leq M .
$$

Using the convexity of $\rho$ and the $\rho$-nonexpansiveness of $T$, we get

$$
\begin{aligned}
\rho\left(\frac{T^{m}\left(x_{k}\right)-x_{k}}{m}\right) & =\rho\left(\frac{1}{m} \sum_{j=0}^{m-1}\left(T^{j+1}\left(x_{k}\right)-T^{j}\left(x_{k}\right)\right)\right) \\
& \leq \frac{1}{m} \sum_{j=0}^{m-1} \rho\left(T^{j+1}\left(x_{k}\right)-T^{j}\left(x_{k}\right)\right) \leq \rho\left(T\left(x_{k}\right)-x_{k}\right)\left(\sum_{j=1}^{m-1} a_{j}\left(x_{n}\right)+1\right) \\
& \leq \frac{1}{m}(M+1) \rho\left(T\left(x_{k}\right)-x_{k}\right) \rightarrow 0,
\end{aligned}
$$

as $k \rightarrow \infty$.

Corollary 4.1 If, under the hypothesis of Lemma 4.1, $\rho$ satisfies additionally the $\Delta_{2}$ condition, then $\rho\left(T^{m}\left(x_{k}\right)-x_{k}\right) \rightarrow 0$ as $k \rightarrow \infty$.

The version of the Demiclosedness Principle used in this paper (Theorem 4.1) requires the uniform continuity of the function modular $\rho$ in the sense of the following definition (see, e.g., [17]).

Definition 4.1 We say that $\rho \in \Re$ is uniformly continuous if to every $\varepsilon>0$ and $L>0$, there exists $\delta>0$ such that

$$
|\rho(g)-\rho(g+h)| \leq \varepsilon
$$

provided $\rho(h)<\delta$ and $\rho(g) \leq L$.

Let us mention that the uniform continuity holds for a large class of function modulars. For instance, it can be proved that in Orlicz spaces over a finite atomless measure [37] or in sequence Orlicz spaces [11] the uniform continuity of the Orlicz modular is equivalent to the $\Delta_{2}$-type condition.

Theorem 4.1 Demiclosedness Principle. Let $\rho \in \Re$. Assume that:

(1) $\rho$ is (UCC1),

(2) $\rho$ has strong Opial property,

(3) $\rho$ has $\Delta_{2}$ property and is uniformly continuous.

Let $C \subset L_{\rho}$ be a nonempty, convex, strongly $\rho$-bounded and $\rho$-closed, and let $T \in \mathcal{T}_{r}(C)$. Let $\left\{x_{n}\right\} \subset C$, and $x \in C$. If $x_{n} \rightarrow x \rho$-a.e. and $\rho\left(T\left(x_{n}\right)-x_{n}\right) \rightarrow 0$, then $x \in F(T)$.

Proof Let us recall that by definition of uniform continuity of $\rho$ to every $\varepsilon>0$ and $L>0$, there exists $\delta>0$ such that

$$
|\rho(g)-\rho(g+h)| \leq \varepsilon
$$


provided $\rho(h)<\delta$ and $\rho(g) \leq L$. Fix any $m \in \mathbb{N}$. Noting that $\rho\left(x_{n}-x\right) \leq M<\infty$ due to the strong $\rho$-boundedness of $C$ and that $\rho\left(T^{m}\left(x_{n}\right)-x_{n}\right) \rightarrow 0$ by Corollary (4.1), it follows from (4.5) with $g=x_{n}-x$ and $h=T^{m}\left(x_{n}\right)-x_{n}$ that

$$
\left|\rho\left(x_{n}-x\right)-\rho\left(x_{n}-x+T^{m}\left(x_{n}\right)-x_{n}\right)\right| \rightarrow 0,
$$

as $n \rightarrow \infty$. Hence

$$
\limsup _{n \rightarrow \infty} \rho\left(x_{n}-x\right)=\limsup _{n \rightarrow \infty} \rho\left(T^{m}\left(x_{n}\right)-x\right) .
$$

Define the $\rho$-type $\varphi$ by

$$
\varphi(x)=\limsup _{n \rightarrow \infty} \rho\left(x_{n}-x\right) .
$$

By (4.7) we get

$$
\varphi(x)=\limsup _{n \rightarrow \infty} \rho\left(T^{m}\left(x_{n}\right)-x\right) .
$$

Hence, for every $y \in C$ there holds

$$
\varphi\left(T^{m}(y)\right)=\limsup _{n \rightarrow \infty} \rho\left(T^{m}\left(x_{n}\right)-T^{m}(y)\right) \leq a_{m}(y) \limsup _{n \rightarrow \infty} \rho\left(x_{n}-y\right)=a_{m}(y) \varphi(y) .
$$

Using (4.10) with $y=x$ and by passing with $m$ to infinity, we conclude that

$$
\limsup _{m \rightarrow \infty} \varphi\left(T^{m}(x)\right) \leq \varphi(x)
$$

Since $\rho$ satisfies the strong Opial property, it also satisfies the Opial property. Since $x_{n} \rightarrow$ $x \rho$-a.e., it follows via the Opial property that for any $y \neq x$

$$
\varphi(x)=\limsup _{n \rightarrow \infty} \rho\left(x_{n}-x\right)<\limsup _{n \rightarrow \infty} \rho\left(x_{n}-y\right)=\varphi(y),
$$

which implies that

$$
\varphi(x)=\inf \{\varphi(y): y \in C\} .
$$

Combining (4.11) with (4.13), we have

$$
\varphi(x) \leq \limsup _{m \rightarrow \infty} \varphi\left(T^{m}(x)\right) \leq \varphi(x),
$$

that is,

$$
\limsup _{m \rightarrow \infty} \varphi\left(T^{m}(x)\right)=\varphi(x) .
$$

We claim that

$$
\lim _{m \rightarrow \infty} \rho\left(T^{m}(x)-x\right)=0 .
$$


Assume to the contrary that (4.16) does not hold, that is,

$$
\rho\left(T^{m}(x)-x\right) \text { does not tend to zero. }
$$

By $\Delta_{2}$, it follows from (4.17) that $\rho\left(\frac{T^{m}(x)-x}{2}\right)$ does not tend to zero. By passing to a subsequence if necessary, we can assume that there exists $0<t<M$ such that

$$
\rho\left(\frac{T^{m}(x)-x}{2}\right)>t>0
$$

for $m \in \mathbb{N}$, which implies that

$$
\rho\left(x_{n}-x\right)+\rho\left(x_{n}-T^{m}(x)\right)>\frac{t}{2},
$$

for every $m, n \in \mathbb{N}$. Hence,

$$
\max \left\{\rho\left(x_{n}-x\right), \rho\left(x_{n}-T^{m}(x)\right)\right\} \geq \frac{t}{4}
$$

for every $m, n \in \mathbb{N}$. Applying the modular parallelogram inequality valid in (UCC1) modular function spaces, see Lemma 4.2 in [18],

$$
\rho^{2}\left(\frac{z+y}{2}\right) \leq \frac{1}{2} \rho^{2}(z)+\frac{1}{2} \rho^{2}(y)-\Psi\left(r, s, \frac{1}{r} \rho(z-y)\right),
$$

where $\rho(z) \leq r, \rho(y) \leq r$ and $\max \{\rho(z), \rho(y)\} \geq s$ for $0<s<r$, with $r=M, s=\frac{t}{4}, z=x_{n}-x$, $y=T^{m}(x)$, we get

$$
\begin{aligned}
\rho^{2}\left(x_{n}-\frac{x+T^{m}(x)}{2}\right) \leq & \frac{1}{2} \rho^{2}\left(x_{n}-x\right)+\frac{1}{2} \rho^{2}\left(x_{n}-T^{m}(x)\right) \\
& -\Psi\left(M, \frac{t}{4}, \frac{1}{M} \rho\left(x-T^{m}(x)\right)\right) .
\end{aligned}
$$

Note that by (4.13)

$$
\varphi^{2}(x) \leq \varphi^{2}\left(\frac{x+T^{m}(x)}{2}\right)=\limsup _{n \rightarrow \infty} \rho^{2}\left(x_{n}-\frac{x+T^{m}(x)}{2}\right) .
$$

Combining (4.22) with (4.23), we obtain

$$
\begin{aligned}
\varphi^{2}(x) \leq & \frac{1}{2} \limsup _{n \rightarrow \infty} \rho^{2}\left(x_{n}-x\right) \\
& +\frac{1}{2} \limsup _{n \rightarrow \infty} \rho^{2}\left(x_{n}-T^{m}(x)\right)-\Psi\left(M, \frac{t}{4}, \frac{1}{M} \rho\left(x-T^{m}(x)\right)\right),
\end{aligned}
$$

which implies

$$
0 \leq \Psi\left(M, \frac{t}{4}, \frac{1}{M} \rho\left(x-T^{m}(x)\right)\right) \leq \frac{1}{2} \varphi^{2}\left(T^{m}(x)\right)-\frac{1}{2} \varphi^{2}(x) .
$$


Letting $m \rightarrow \infty$ and applying (4.15), we get

$$
\begin{aligned}
0 & \leq \limsup _{m \rightarrow \infty} \Psi\left(M, \frac{t}{4}, \frac{1}{M} \rho\left(x-T^{m}(x)\right)\right) \\
& \leq \frac{1}{2} \limsup _{m \rightarrow \infty} \varphi^{2}\left(T^{m}(x)\right)-\frac{1}{2} \varphi^{2}(x) \leq 0 .
\end{aligned}
$$

Using the properties of $\Psi$, we conclude that $\rho\left(x-T^{m}(x)\right)$ tends to zero itself, which contradicts our assumption (4.17). Hence, $\rho\left(x-T^{m}(x)\right) \rightarrow 0$ as $m \rightarrow \infty$. Clearly, then $\rho\left(x-T^{m+1}(x)\right) \rightarrow 0$ as $m \rightarrow \infty$, that is, $T^{m+1}(x) \rightarrow x(\rho)$ while $T^{m+1}(x) \rightarrow T(x)(\rho)$ by $\rho-$ continuity of $T$. By the uniqueness of the $\rho$-limit, we obtain $T(x)=x$, that is, $x \in F(T)$.

\section{Convergence of generalized Mann iteration process}

The following elementary, easy to prove, lemma will be used in this paper.

Lemma 5.1 [2] Suppose $\left\{r_{k}\right\}$ is a bounded sequence of real numbers and $\left\{d_{k, n}\right\}$ is a doublyindex sequence of real numbers which satisfy

$$
\limsup _{k \rightarrow \infty} \limsup _{n \rightarrow \infty} d_{k, n} \leq 0 \text { and } r_{k+n} \leq r_{k}+d_{k, n}
$$

for each $k, n \geq 1$. Then $\left\{r_{k}\right\}$ converges to an $r \in \mathbb{R}$.

Following Mann [29], let us start with the definition of the generalized Mann iteration process.

Definition 5.1 Let $T \in \mathcal{T}_{r}(C)$ and let $\left\{n_{k}\right\}$ be an increasing sequence of natural numbers. Let $\left\{t_{k}\right\} \subset(0,1)$ be bounded away from 0 and 1 . The generalized Mann iteration process generated by the mapping $T$, the sequence $\left\{t_{k}\right\}$, and the sequence $\left\{n_{k}\right\}$ denoted by $g M\left(T,\left\{t_{k}\right\},\left\{n_{k}\right\}\right)$ is defined by the following iterative formula:

$$
x_{k+1}=t_{k} T^{n_{k}}\left(x_{k}\right)+\left(1-t_{k}\right) x_{k}, \quad \text { where } x_{1} \in C \text { is chosen arbitrarily. }
$$

Definition 5.2 We say that a generalized Mann iteration process $g M\left(T,\left\{t_{k}\right\},\left\{n_{k}\right\}\right)$ is well defined if

$$
\limsup _{k \rightarrow \infty} a_{n_{k}}\left(x_{k}\right)=1 \text {. }
$$

Remark 5.1 Observe that by the definition of asymptotic pointwise nonexpansiveness, $\lim _{k \rightarrow \infty} a_{k}(x)=1$ for every $x \in C$. Hence we can always select a subsequence $\left\{a_{n_{k}}\right\}$ such that (5.2) holds. In other words, by a suitable choice of $\left\{n_{k}\right\}$, we can always make $g M\left(T,\left\{t_{k}\right\},\left\{n_{k}\right\}\right)$ well defined.

The following result provides an important technique which will be used in this paper.

Lemma 5.2 Let $\rho \in \Re$ be (UUC1). Let $C \subset L_{\rho}$ be a $\rho$-closed, $\rho$-bounded and convex set. Let $T \in \mathcal{T}_{r}(C)$ and let $\left\{n_{k}\right\} \subset \mathbb{N}$. Assume that a sequence $\left\{t_{k}\right\} \subset(0,1)$ is bounded away from 
0 and 1. Let $w$ be a fixed point of $T$ and $g M\left(T,\left\{t_{k}\right\},\left\{n_{k}\right\}\right)$ be a generalized Mann process. Then there exists $r \in \mathbb{R}$ such that

$$
\lim _{k \rightarrow \infty} \rho\left(x_{k}-w\right)=r
$$

Proof Let $w \in F(T)$. Since

$$
\begin{aligned}
\rho\left(x_{k+1}-w\right) & \leq t_{k} \rho\left(T^{n_{k}}\left(x_{k}\right)-w\right)+\left(1-t_{k}\right) \rho\left(x_{k}-w\right) \\
& =t_{k} \rho\left(T^{n_{k}}\left(x_{k}\right)-T^{n_{k}}(w)\right)+\left(1-t_{k}\right) \rho\left(x_{k}-w\right) \\
& \leq t_{k}\left(1+b_{n_{k}}(w)\right) \rho\left(x_{k}-w\right)+\left(1-t_{k}\right) \rho\left(x_{k}-w\right) \\
& =t_{k} b_{n_{k}}(w) \rho\left(x_{k}-w\right)+\rho\left(x_{k}-w\right) \\
& \leq b_{n_{k}}(w) \operatorname{diam}_{\rho}(C)+\rho\left(x_{k}-w\right),
\end{aligned}
$$

it follows that for every $n \in \mathbb{N}$,

$$
\rho\left(x_{k+n}-w\right) \leq \rho\left(x_{k}-w\right)+\operatorname{diam}_{\rho}(C) \sum_{i=k}^{k+n-1} b_{n_{i}}(w) .
$$

Denote $r_{p}=\rho\left(x_{p}-w\right)$ for every $p \in \mathbb{N}$ and $d_{k, n}=\operatorname{diam}_{\rho}(C) \sum_{i=k}^{k+n-1} b_{n_{i}}(w)$. Observe that since $T \in \mathcal{T}_{r}(C)$, it follows that limsup $\sup _{k \rightarrow \infty} \lim \sup _{n \rightarrow \infty} d_{k, n}=0$. By Lemma 5.1, there exists an $r \in \mathbb{R}$ such that $\lim _{k \rightarrow \infty} \rho\left(x_{k}-w\right)=r$ as claimed.

The next result will be essential for proving the convergence theorems for iterative process.

Lemma 5.3 Let $\rho \in \Re$ be (UUC1). Let $C \subset L_{\rho}$ be a $\rho$-closed, $\rho$-bounded and convex set, and $T \in \mathcal{T}_{r}(C)$. Assume that a sequence $\left\{t_{k}\right\} \subset(0,1)$ is bounded away from 0 and 1 . Let $\left\{n_{k}\right\} \subset \mathbb{N}$ and $g M\left(T,\left\{t_{k}\right\},\left\{n_{k}\right\}\right)$ be a generalized Mann iteration process. Then

$$
\lim _{k \rightarrow \infty} \rho\left(T^{n_{k}}\left(x_{k}\right)-x_{k}\right)=0,
$$

and

$$
\lim _{k \rightarrow \infty} \rho\left(x_{k+1}-x_{k}\right)=0
$$

Proof By Theorem 3.1, $T$ has at least one fixed point $w \in C$. In view of Lemma 5.2, there exists $r \in \mathbb{R}$ such that

$$
\lim _{k \rightarrow \infty} \rho\left(x_{k}-w\right)=r
$$

Note that

$$
\begin{aligned}
\limsup _{k \rightarrow \infty} \rho\left(T^{n_{k}}\left(x_{k}\right)-w\right) & =\limsup _{k \rightarrow \infty} \rho\left(T^{n_{k}}\left(x_{k}\right)-T^{n_{k}}(w)\right) \\
& \leq \limsup _{k \rightarrow \infty} a_{n_{k}}(w) \rho\left(x_{k}-w\right) \leq r,
\end{aligned}
$$


and that

$$
\lim _{k \rightarrow \infty} \rho\left(t_{k}\left(T^{n_{k}}\left(x_{k}\right)-w\right)+\left(1-t_{k}\right)\left(x_{k}-w\right)\right)=\lim _{k \rightarrow \infty} \rho\left(x_{k+1}-w\right)=r .
$$

Set $f_{k}=T^{n_{k}}\left(x_{k}\right)-w, g_{k}=x_{k}-w$, and note that $\lim \sup _{k \rightarrow \infty} \rho\left(g_{k}\right) \leq r$ by (5.7), and $\limsup _{k \rightarrow \infty} \rho\left(f_{k}\right) \leq r$ by (5.8). Observe also that

$$
\begin{aligned}
& \lim _{k \rightarrow \infty} \rho\left(t_{k} f_{k}+\left(1-t_{k}\right) g_{k}\right) \\
& \quad=\lim _{k \rightarrow \infty} \rho\left(t_{k} T^{n_{k}}\left(x_{k}\right)+\left(1-t_{k}\right) x_{k}-w\right) \\
& \quad=\lim _{k \rightarrow \infty} \rho\left(x_{k+1}-w\right)=r .
\end{aligned}
$$

Hence, it follows from Lemma 3.2 that

$$
\lim _{k \rightarrow \infty} \rho\left(T^{n_{k}}\left(x_{k}\right)-x_{k}\right)=\lim _{k \rightarrow \infty} \rho\left(f_{k}-g_{k}\right)=0,
$$

which by the construction of the sequence $\left\{x_{k}\right\}$ is equivalent to

$$
\lim _{k \rightarrow \infty} \rho\left(x_{k+1}-x_{k}\right)=0
$$

as claimed.

In the next lemma, we prove that under suitable assumption the sequence $\left\{x_{k}\right\}$ becomes an approximate fixed point sequence, which will provide an important step in the proof of the generalized Mann iteration process convergence. First, we need to recall the following notions.

Definition 5.3 A strictly increasing sequence $\left\{n_{i}\right\} \subset \mathbb{N}$ is called quasi-periodic if the sequence $\left\{n_{i+1}-n_{i}\right\}$ is bounded, or equivalently, if there exists a number $p \in \mathbb{N}$ such that any block of $p$ consecutive natural numbers must contain a term of the sequence $\left\{n_{i}\right\}$. The smallest of such numbers $p$ will be called a quasi-period of $\left\{n_{i}\right\}$.

Lemma 5.4 Let $\rho \in \Re$ be (UUC1) satisfying $\Delta_{2}$. Let $C \subset L_{\rho}$ be a $\rho$-closed, $\rho$-bounded and convex set, and $T \in \mathcal{T}_{r}(C)$. Let $\left\{t_{k}\right\} \subset(0,1)$ be bounded away from 0 and 1 . Let $\left\{n_{k}\right\} \subset \mathbb{N}$ be such that the generalized Mann process $g M\left(T,\left\{t_{k}\right\},\left\{n_{k}\right\}\right)$ is well defined. If, in addition, the set of indices $\mathcal{J}=\left\{j ; n_{j+1}=1+n_{j}\right\}$ is quasi-periodic, then $\left\{x_{k}\right\}$ is an approximate fixed point sequence, i.e.,

$$
\lim _{k \rightarrow \infty} \rho\left(T\left(x_{k}\right)-x_{k}\right)=0 .
$$

Proof Let $p \in \mathbb{N}$ be a quasi-period of $\mathcal{J}$. Observe that it is enough to prove that $\rho\left(T\left(x_{k}\right)-\right.$ $\left.x_{k}\right) \rightarrow 0$ as $k \rightarrow \infty$ through $\mathcal{J}$. Indeed, let us fix $\varepsilon>0$. From $\rho\left(T\left(x_{k}\right)-x_{k}\right) \rightarrow 0$ as $k \rightarrow \infty$ through $\mathcal{J}$ it follows that

$$
\rho\left(T\left(x_{k}\right)-x_{k}\right)<\varepsilon
$$


for sufficiently large $k$. By the quasi-periodicity of $\mathcal{J}$, to every positive integer $k$, there exists $j_{k} \in \mathcal{J}$ such that $\left|k-j_{k}\right| \leq p$. Assume that $k-p \leq j_{k} \leq k$ (the proof for the other case is identical). Since $T$ is $\rho$-Lipschitzian with the constant $M=\sup \left\{a_{1}(x) ; x \in C\right\}$, there exist a $0<\delta<\frac{\varepsilon}{3}$ such that

$$
\rho(T(x)-T(y))<\varepsilon \quad \text { if } \rho(x-y)<\delta .
$$

Note that by (5.6) and by $\Delta_{2}, \rho\left(p\left(x_{k+1}-x_{k}\right)\right)<\frac{\delta}{p}$ for $k$ sufficiently large. This implies that

$$
\rho\left(x_{k}-x_{j_{k}}\right) \leq \frac{1}{p}\left(\rho\left(p\left(x_{k}-x_{k-1}\right)\right)+\cdots+\rho\left(p\left(x_{j_{k}+1}-x_{j_{k}}\right)\right)\right) \leq p \frac{\delta}{p}=\delta,
$$

and therefore,

$$
\begin{aligned}
\rho\left(\frac{x_{k}-T\left(x_{k}\right)}{3}\right) & \leq \frac{1}{3} \rho\left(x_{k}-x_{j_{k}}\right)+\frac{1}{3} \rho\left(x_{j_{k}}-T\left(x_{j_{k}}\right)\right)+\frac{1}{3} \rho\left(T\left(x_{j_{k}}\right)-T\left(x_{k}\right)\right) \\
& \leq \delta+\frac{\varepsilon}{3}+\frac{\varepsilon}{3}<\varepsilon,
\end{aligned}
$$

which demonstrates that

$$
\rho\left(\frac{x_{k}-T\left(x_{k}\right)}{3}\right) \rightarrow 0
$$

as $k \rightarrow \infty$. By $\Delta_{2}$ again, we get $\rho\left(T\left(x_{k}\right)-x_{k}\right) \rightarrow \infty$.

To prove that $\rho\left(T\left(x_{k}\right)-x_{k}\right) \rightarrow 0$ as $k \rightarrow \infty$ through $\mathcal{J}$, observe that, since $n_{k+1}=n_{k}+1$ for such $k$, there holds

$$
\begin{aligned}
\rho\left(\frac{x_{k}-T\left(x_{k}\right)}{4}\right) \leq & \frac{1}{4} \rho\left(x_{k}-x_{k+1}\right)+\frac{1}{4} \rho\left(x_{k+1}-T^{n_{k+1}}\left(x_{k+1}\right)\right) \\
& +\frac{1}{4} \rho\left(T^{n_{k+1}}\left(x_{k+1}\right)-T^{n_{k+1}}\left(x_{k}\right)\right)+\frac{1}{4} \rho\left(T T^{n_{k}}\left(x_{k}\right)-T\left(x_{k}\right)\right) \\
\leq & \frac{1}{4} \rho\left(x_{k}-x_{k+1}\right)+\frac{1}{4} \rho\left(x_{k+1}-T^{n_{k+1}}\left(x_{k+1}\right)\right) \\
& +\frac{1}{4} a_{n_{k+1}}\left(x_{k+1}\right) \rho\left(x_{k}-x_{k+1}\right)+\frac{1}{4} M \rho\left(T^{n_{k}}\left(x_{k}\right)-x_{k}\right)
\end{aligned}
$$

which tends to zero in view of (5.5), (5.6) and (5.2).

The next theorem is the main result of this section.

Theorem 5.1 Let $\rho \in \Re$. Assume that:

(1) $\rho$ is (UCC1),

(2) $\rho$ has Strong Opial Property,

(3) $\rho$ has $\Delta_{2}$ property and is uniformly continuous.

Let $C \subset L_{\rho}$ be nonempty, $\rho$-a.e. compact, convex, strongly $\rho$-bounded and $\rho$-closed, and let $T \in \mathcal{T}_{r}(C)$. Assume that a sequence $\left\{t_{k}\right\} \subset(0,1)$ is bounded away from 0 and 1 . Let $\left\{n_{k}\right\} \subset$ $\mathbb{N}$ and $g M\left(T,\left\{t_{k}\right\},\left\{n_{k}\right\}\right)$ be a well-defined generalized Mann iteration process. Assume, in addition, that the set of indices $\mathcal{J}=\left\{j ; n_{j+1}=1+n_{j}\right\}$ is quasi-periodic. Then there exists $x \in F(T)$ such that $x_{n} \rightarrow x \rho$-a.e. 
Proof Observe that by Theorem 4.1 in [18], the set of fixed points $F(T)$ is nonempty, convex and $\rho$-closed. Note also that by Lemma 3.1 in [27], it follows from the strong Opial property of $\rho$ that any $\rho$-type attains its minimum in C. By Lemma 5.4, the sequence $\left\{x_{k}\right\}$ is an approximate fixed point sequence, that is,

$$
\rho\left(T\left(x_{k}\right)-x_{k}\right) \rightarrow 0
$$

as $k \rightarrow \infty$. Consider $y, z \in C$, two $\rho$-a.e. cluster points of $\left\{x_{k}\right\}$. There exits then $\left\{y_{k}\right\},\left\{z_{k}\right\}$ subsequences of $\left\{x_{k}\right\}$ such that $y_{k} \rightarrow y \rho$-a.e. and $z_{k} \rightarrow z \rho$-a.e. By Theorem 4.1, $y \in F(T)$ and $z \in F(T)$. By Lemma 5.2, there exist $r_{y}, r_{z} \in \mathbb{R}$ such that

$$
r_{y}=\lim _{k \rightarrow} \rho\left(x_{k}-y\right), \quad r_{z}=\lim _{k \rightarrow} \rho\left(x_{k}-z\right)
$$

We claim that $y=z$. Assume to the contrary that $y \neq z$. Then, by the strong Opial property, we have

$$
\begin{aligned}
r_{y} & =\liminf _{k \rightarrow \infty} \rho\left(y_{k}-y\right)<\liminf _{k \rightarrow \infty} \rho\left(y_{k}-z\right) \\
& =\liminf _{k \rightarrow \infty} \rho\left(z_{k}-z\right)<\liminf _{k \rightarrow \infty} \rho\left(z_{k}-y\right)=r_{y} .
\end{aligned}
$$

The contradiction implies that $y=z$. Therefore, $\left\{x_{k}\right\}$ has at most one $\rho$-a.e. cluster point. Since, $C$ is $\rho$-a.e. compact it follows that the sequence $\left\{x_{k}\right\}$ has exactly one $\rho$-a.e. cluster point, which means that $\rho\left(x_{k}\right) \rightarrow x \rho$-a.e. Using Theorem 4.1 again, we get $x \in F(T)$ as claimed.

Remark 5.2 It is easy to see that we can always construct a sequence $\left\{n_{k}\right\}$ with the quasiperiodic properties specified in the assumptions of Theorem 5.1. When constructing concrete implementations of this algorithm, the difficulty will be to ensure that the constructed sequence $\left\{n_{k}\right\}$ is not "too sparse" in the sense that the generalized Mann process $g M\left(T,\left\{t_{k}\right\},\left\{n_{k}\right\}\right)$ remains well defined. The similar quasi-periodic type assumptions are common in the asymptotic fixed point theory, see, e.g., $[2,25,28]$.

\section{Convergence of generalized Ishikawa iteration process}

The two-step Ishikawa iteration process is a generalization of the one-step Mann process. The Ishikawa iteration process, [10], provides more flexibility in defining the algorithm parameters, which is important from the numerical implementation perspective.

Definition 6.1 Let $T \in \mathcal{T}_{r}(C)$ and let $\left\{n_{k}\right\}$ be an increasing sequence of natural numbers. Let $\left\{t_{k}\right\} \subset(0,1)$ be bounded away from 0 and 1 , and $\left\{s_{k}\right\} \subset(0,1)$ be bounded away from 1 . The generalized Ishikawa iteration process generated by the mapping $T$, the sequences $\left\{t_{k}\right\},\left\{s_{k}\right\}$, and the sequence $\left\{n_{k}\right\}$ denoted by $g I\left(T,\left\{t_{k}\right\},\left\{s_{k}\right\},\left\{n_{k}\right\}\right)$ is defined by the following iterative formula:

$$
x_{k+1}=t_{k} T^{n_{k}}\left(s_{k} T^{n_{k}}\left(x_{k}\right)+\left(1-s_{k}\right) x_{k}\right)+\left(1-t_{k}\right) x_{k},
$$

where $x_{1} \in C$ is chosen arbitrarily. 
Definition 6.2 We say that a generalized Ishikawa iteration process $g I\left(T,\left\{t_{k}\right\},\left\{s_{k}\right\},\left\{n_{k}\right\}\right)$ is well defined if

$$
\limsup _{k \rightarrow \infty} a_{n_{k}}\left(x_{k}\right)=1
$$

Remark 6.1 Observe that, by the definition of asymptotic pointwise nonexpansiveness, $\lim _{k \rightarrow \infty} a_{k}(x)=1$ for every $x \in C$. Hence we can always select a subsequence $\left\{a_{n_{k}}\right\}$ such that (6.2) holds. In other words, by a suitable choice of $\left\{n_{k}\right\}$, we can always make $g I\left(T,\left\{t_{k}\right\},\left\{s_{k}\right\},\left\{n_{k}\right\}\right)$ well defined.

Lemma 6.1 Let $\rho \in \Re$ be (UUC1). Let $C \subset L_{\rho}$ be a $\rho$-closed, $\rho$-bounded and convex set. Let $T \in \mathcal{T}_{r}(C)$ and let $\left\{n_{k}\right\} \subset \mathbb{N}$. Let $\left\{t_{k}\right\} \subset(0,1)$ be bounded away from 0 and 1 , and $\left\{s_{k}\right\} \subset(0,1)$ be bounded away from 1 . Let $w \in F(T)$ and $g I\left(T,\left\{t_{k}\right\},\left\{s_{k}\right\},\left\{n_{k}\right\}\right)$ be a generalized Ishikawa process. There exists then an $r \in \mathbb{R}$ such that $\lim _{k \rightarrow \infty} \rho\left(x_{k}-w\right)=r$.

Proof Define $T_{k}: C \rightarrow C$ by

$$
T_{k}(x)=t_{k} T^{n_{k}}\left(s_{k} T^{n_{k}}(x)+\left(1-s_{k}\right) x\right)+\left(1-t_{k}\right) x, \quad x \in C .
$$

It is easy to see that $x_{k+1}=T_{k}\left(x_{k}\right)$ and that $F(T) \subset F\left(T_{k}\right)$. Moreover, a straight calculation shows that each $T_{k}$ satisfies

$$
\rho\left(T_{k}(x)-T_{k}(y)\right) \leq A_{k}(x) \rho(x-y)
$$

where

$$
A_{k}(x)=1+t_{k} a_{n_{k}}\left(M_{k}(x)\right)\left(1+s_{k} a_{n_{k}}(x)-s_{k}\right)-t_{k},
$$

and

$$
M_{k}(x)=s_{k} T^{n_{k}}(x)+\left(1-s_{k}\right) x .
$$

Note that $A_{k}(x) \geq 1$, which follows directly from the fact that $a_{n_{k}}(x) \geq 1$ and from (6.5). Using (6.5) and the fact that $M_{k}(w)=w$, we have

$$
B_{k}(w)=A_{k}(w)-1=t_{k}\left(1+s_{k} a_{n_{k}}(w)\right)\left(a_{n_{k}}(w)-1\right) \leq\left(1+a_{n_{k}}(w)\right) b_{n_{k}}(w) .
$$

Fix any $M>1$. Since $\lim _{k \rightarrow \infty} a_{n_{k}}(w)=1$, it follows that there exists a $k_{0} \geq 1$ such that for $k>k_{0}, a_{n_{k}}(w) \leq M$. Therefore, using the same argument as in the proof of Lemma 5.2, we deduce that for $k>k_{0}$ and $n>1$

$$
\begin{aligned}
\rho\left(x_{k+n}-w\right) & \leq \rho\left(x_{k}-w\right)+\operatorname{diam}_{\rho}(C) \sum_{i=k}^{k+n-1} B_{i}(w) \\
& \leq \rho\left(x_{k}-w\right)+\operatorname{diam}_{\rho}(C)(1+M) \sum_{i=k}^{k+n-1} b_{n_{i}}(w) .
\end{aligned}
$$


Arguing like in the proof of Lemma 5.2, we conclude that there exists an $r \in \mathbb{R}$ such that $\lim _{k \rightarrow \infty} \rho\left(x_{k}-w\right)=r$.

Lemma 6.2 Let $\rho \in \Re$ be (UUC1). Let $C \subset L_{\rho}$ be a $\rho$-closed, $\rho$-bounded and convex set. Let $T \in \mathcal{T}_{r}(C)$ and let $\left\{n_{k}\right\} \subset \mathbb{N}$. Let $\left\{t_{k}\right\} \subset(0,1)$ be bounded away from 0 and 1 , and $\left\{s_{k}\right\} \subset(0,1)$ be bounded away from 1 . Let $\mathrm{gI}\left(T,\left\{t_{k}\right\},\left\{s_{k}\right\},\left\{n_{k}\right\}\right)$ be a generalized Ishikawa process. Define

$$
y_{k}=s_{k} T^{n_{k}}\left(x_{k}\right)+\left(1-s_{k}\right) x_{k} .
$$

Then

$$
\lim _{k \rightarrow \infty} \rho\left(T^{n_{k}}\left(y_{k}\right)-x_{k}\right)=0,
$$

or equivalently

$$
\lim _{k \rightarrow \infty} \rho\left(x_{k+1}-x_{k}\right)=0 .
$$

Proof By Theorem 3.1, $F(T) \neq \emptyset$. Let us fix $w \in F(T)$. By Lemma 6.1, $\lim _{k \rightarrow \infty} \rho\left(x_{k}-w\right)$ exists. Let us denote it by $r$. Since $w \in F(T), T \in \mathcal{T}_{r}(C)$, and $\lim _{k \rightarrow \infty} \rho\left(x_{k}-w\right)=r$ by Lemma 6.1, we have the following:

$$
\begin{aligned}
& \limsup _{k \rightarrow \infty} \rho\left(T^{n_{k}}\left(y_{k}\right)-w\right) \\
& \quad=\limsup _{k \rightarrow \infty} \rho\left(T^{n_{k}}\left(y_{k}\right)-T^{n_{k}}(w)\right) \\
& \quad \leq \limsup _{k \rightarrow \infty} a_{n_{k}}(w) \rho\left(y_{k}-w\right)=\limsup _{k \rightarrow \infty} a_{n_{k}}(w) \rho\left(s_{k} T^{n_{k}}\left(x_{k}\right)+\left(1-s_{k}\right) x_{k}-w\right) \\
& \quad \leq \limsup _{k \rightarrow \infty}\left(s_{k} a_{n_{k}}(w) \rho\left(T^{n_{k}}\left(x_{k}\right)-w\right)+\left(1-s_{k}\right) a_{n_{k}}(w) \rho\left(x_{k}-w\right)\right) \\
& \quad \leq \limsup _{k \rightarrow \infty}\left(s_{k} a_{n_{k}}^{2}(w) \rho\left(x_{k}-w\right)+\left(1-s_{k}\right) a_{n_{k}}(w) \rho\left(x_{k}-w\right)\right) \leq r .
\end{aligned}
$$

Note that

$$
\begin{aligned}
& \lim _{k \rightarrow \infty} \rho\left(t_{k}\left(T^{n_{k}}\left(y_{k}\right)-w\right)+\left(1-t_{k}\right)\left(x_{k}-w\right)\right) \\
& \quad=\lim _{k \rightarrow \infty} \rho\left(t_{k} T^{n_{k}}\left(y_{k}\right)+\left(1-t_{k}\right) x_{k}-w\right)=\lim _{k \rightarrow \infty} \rho\left(x_{k+1}-w\right)=r .
\end{aligned}
$$

Applying Lemma 3.2 with $u_{k}=T^{n_{k}}\left(y_{k}\right)-w$ and $v_{k}=x_{k}-w$, we obtain the desired equality $\lim _{k \rightarrow \infty} \rho\left(T^{n_{k}}\left(y_{k}\right)-x_{k}\right)=0$, while (6.11) follows from (6.10) via the construction formulas for $x_{k+1}$ and $y_{k}$.

Lemma 6.3 Let $\rho \in \Re$ be (UUC1) satisfying $\Delta_{2}$. Let $C \subset L_{\rho}$ be a $\rho$-closed, $\rho$-bounded and convex set. Let $T \in \mathcal{T}_{r}(C)$ and let $\left\{n_{k}\right\} \subset \mathbb{N}$. Let $\left\{t_{k}\right\} \subset(0,1)$ be bounded away from 0 and 1 , and $\left\{s_{k}\right\} \subset(0,1)$ be bounded away from 1 . Let $g I\left(T,\left\{t_{k}\right\},\left\{s_{k}\right\},\left\{n_{k}\right\}\right)$ be a well-defined generalized Ishikawa process. Then

$$
\lim _{k \rightarrow \infty} \rho\left(T^{n_{k}}\left(x_{k}\right)-x_{k}\right)=0 .
$$


Proof Let $y_{k}=s_{k} T^{n_{k}}\left(x_{k}\right)+\left(1-s_{k}\right) x_{k}$. Hence

$$
T^{n_{k}}\left(x_{k}\right)-x_{k}=\frac{1}{1-s_{k}}\left(T^{n_{k}}\left(x_{k}\right)-y_{k}\right) .
$$

Since $\left\{s_{k}\right\} \subset(0,1)$ is bounded away from 1 , there exists $0<s<1$ such that $s_{k} \leq s$ for every $k \geq 1$. Hence,

$$
\rho\left(T^{n_{k}}\left(x_{k}\right)-x_{k}\right)=\rho\left(\frac{1}{1-s_{k}}\left(T^{n_{k}}\left(x_{k}\right)-y_{k}\right)\right) \leq \rho\left(\frac{1}{1-s}\left(T^{n_{k}}\left(x_{k}\right)-y_{k}\right)\right) .
$$

The right-hand side of this inequality tends to zero because $\rho\left(T^{n_{k}}\left(x_{k}\right)-y_{k}\right) \rightarrow 0$ by Lemma 6.2 and $\rho$ satisfies $\Delta_{2}$.

Lemma 6.4 Let $\rho \in \Re$ be (UUC1) satisfying $\Delta_{2}$. Let $C \subset L_{\rho}$ be a $\rho$-closed, $\rho$-bounded and convex set, and $T \in \mathcal{T}_{r}(C)$. Let $\left\{t_{k}\right\} \subset(0,1)$ be bounded away from 0 and 1 and $\left\{s_{k}\right\} \subset$ $(0,1)$ be bounded away from 1 . Let $\left\{n_{k}\right\} \subset \mathbb{N}$ be such that the generalized Ishikawa process $g I\left(T,\left\{t_{k}\right\},\left\{s_{k}\right\},\left\{n_{k}\right\}\right)$ is well defined. If, in addition, the set $\mathcal{J}=\left\{j ; n_{j+1}=1+n_{j}\right\}$ is quasiperiodic, then $\left\{x_{k}\right\}$ is an approximate fixed point sequence, i.e.,

$$
\lim _{k \rightarrow \infty} \rho\left(T\left(x_{k}\right)-x_{k}\right)=0
$$

Proof The proof is analogous to that of Lemma 5.4 with (6.11) used instead of (5.6) and (6.14) replacing (5.5).

Theorem 6.1 Let $\rho \in \Re$. Assume that

(1) $\rho$ is (UCC1),

(2) $\rho$ has Strong Opial Property,

(3) $\rho$ has $\Delta_{2}$ property and is uniformly continuous.

Let $C \subset L_{\rho}$ be nonempty, $\rho$-a.e. compact, convex, strongly $\rho$-bounded and $\rho$-closed, and let $T \in \mathcal{T}_{r}(C)$. Let $T \in \mathcal{T}_{r}(C)$. Let $\left\{t_{k}\right\} \subset(0,1)$ be bounded away from 0 and 1 , and $\left\{s_{k}\right\} \subset(0,1)$ be bounded away from 1. Let $\left\{n_{k}\right\}$ be such that the generalized Ishikawa process $g I\left(T,\left\{t_{k}\right\},\left\{s_{k}\right\},\left\{n_{k}\right\}\right)$ is well defined. If, in addition, the set $\mathcal{J}=\left\{j ; n_{j+1}=1+n_{j}\right\}$ is quasi-periodic, then $\left\{x_{k}\right\}$ generated by $g I\left(T,\left\{t_{k}\right\},\left\{s_{k}\right\},\left\{n_{k}\right\}\right)$ converges $\rho$-a.e. to a fixed point $x \in F(T)$.

Proof The proof is analogous to that of Theorem 5.1 with Lemma 5.4 replaced by Lemma 6.4, and Lemma 5.2 replaced by Lemma 6.1.

\section{Strong convergence}

It is interesting that, provided $C$ is $\rho$-compact, both generalized Mann and Ishikawa processes converge strongly to a fixed point of $T$ even without assuming the Opial property.

Theorem 7.1 Let $\rho \in \Re$ satisfy conditions (UUC1) and $\Delta_{2}$. Let $C \subset L_{\rho}$ be a $\rho$-compact, $\rho$ bounded and convex set, and let $T \in \mathcal{T}_{r}(C)$. Let $\left\{t_{k}\right\} \subset(0,1)$ be bounded away from 0 and 1 , and $\left\{s_{k}\right\} \subset(0,1)$ be bounded away from 1 . Let $\left\{n_{k}\right\}$ be such that the generalized Mann process $g M\left(T,\left\{t_{k}\right\},\left\{n_{k}\right\}\right)$ (resp. Ishikawa process $\left.g I\left(T,\left\{t_{k}\right\},\left\{s_{k}\right\},\left\{n_{k}\right\}\right)\right)$ is well defined. Then 
there exists a fixed point $x \in F(T)$ such that then $\left\{x_{k}\right\}$ generated by $g M\left(T,\left\{t_{k}\right\},\left\{n_{k}\right\}\right)$ (resp. $\left.g I\left(T,\left\{t_{k}\right\},\left\{s_{k}\right\},\left\{n_{k}\right\}\right)\right)$ converges strongly to a fixed point of $T$, that is

$$
\lim _{k \rightarrow \infty} \rho\left(x_{k}-x\right)=0
$$

Proof By the $\rho$-compactness of $C$, we can select a subsequence $\left\{x_{p_{k}}\right\}$ of $\left\{x_{k}\right\}$ such that there exists $x \in C$ with

$$
\lim _{k \rightarrow \infty} \rho\left(T\left(x_{p_{k}}\right)-x\right)=0
$$

Note that

$$
\rho\left(\frac{x_{p_{k}}-x}{2}\right) \leq \frac{1}{2} \rho\left(x_{p_{k}}-T\left(x_{p_{k}}\right)\right)+\frac{1}{2} \rho\left(T\left(x_{p_{k}}-x\right)\right)
$$

which tends to zero by Lemma 5.3 (resp. Lemma 6.4) and by (7.2). By $\Delta_{2}$ it follows from (7.3) that

$$
\rho\left(x_{p_{k}}-x\right) \rightarrow 0 \quad \text { as } k \rightarrow \infty
$$

Observe that by the convexity of $\rho$ and by $\rho$-nonexpansiveness of $T$, we have

$$
\begin{aligned}
\rho\left(\frac{T(x)-x}{3}\right) & \leq \frac{1}{3} \rho\left(T(x)-T\left(x_{p_{k}}\right)\right)+\frac{1}{3} \rho\left(T\left(x_{p_{k}}\right)-x_{p_{k}}\right)+\frac{1}{3} \rho\left(x_{p_{k}}-x\right) \\
& \leq \frac{1}{3} \rho\left(x-x_{p_{k}}\right)+\frac{1}{3} \rho\left(T\left(x_{p_{k}}\right)-x_{p_{k}}\right)+\frac{1}{3} \rho\left(x_{p_{k}}-x\right)
\end{aligned}
$$

which tends to zero by (7.4) and by Lemma 5.3 (resp. Lemma 6.4). Hence $\rho(T(x)-x)=0$ which implies that $x \in F(T)$. Applying Lemma 5.2 (resp. Lemma 6.1), we conclude that $\lim _{k \rightarrow \infty} \rho\left(x_{k}-x\right)$ exists. By (7.4) this limit must be equal to zero which implies that

$$
\lim _{k \rightarrow \infty} \rho\left(x_{k}-x\right)=0
$$

Remark 7.1 Observe that in view of the $\Delta_{2}$ assumption, the $\rho$-compactness of the set $C$ assumed in Theorem 7.1 is equivalent to the compactness in the sense of the norm defined by $\rho$.

Competing interests

The authors declare that they have no competing interests.

Authors' contributions

Both authors equally participated in all stages of preparations of this article. Both authors read and approved the final manuscript.

\section{Author details}

${ }^{1}$ Department of Mathematics, King Abdulaziz University, P.O. Box 53909, Jeddah 21593, Saudi Arabia. ${ }^{2}$ School of Mathematics and Statistics, University of New South Wales, Sydney, NSW 2052, Australia. 


\section{Acknowledgements}

The authors would like to thank MA Khamsi for his valuable suggestions to improve the presentation of the paper.

\section{Received: 4 April 2012 Accepted: 2 July 2012 Published: 20 July 2012}

\section{References}

1. Bose, SC: Weak convergence to the fixed point of an asymptotically nonexpansive. Proc. Am. Math. Soc. 68, 305-308 (1978)

2. Bruck, R, Kuczumow, T, Reich, S: Convergence of iterates of asymptotically nonexpansive mappings in Banach spaces with the uniform Opial property. Colloq. Math. 65(2), 169-179 (1993)

3. Dominguez-Benavides, T, Khamsi, MA, Samadi, S: Uniformly Lipschitzian mappings in modular function spaces. Nonlinear Anal. 46, 267-278 (2001)

4. Dominguez-Benavides, T, Khamsi, MA, Samadi, S: Asymptotically regular mappings in modular function spaces. Sci. Math. Jpn. 53, 295-304 (2001)

5. Dominguez-Benavides, T, Khamsi, MA, Samadi, S: Asymptotically nonexpansive mappings in modular function spaces. J. Math. Anal. Appl. 265(2), 249-263 (2002)

6. Fukhar-ud-din, H, Khan, AR: Approximating common fixed points of asymptotically nonexpansive maps in uniformly convex Banach spaces. Comput. Math. Appl. 53, 1349-1360 (2009)

7. Gornicki, J: Weak convergence theorems for asymptotically nonexpansive mappings in uniformly convex Banach spaces. Comment. Math. Univ. Carol. 30, 249-252 (1989)

8. Hajji, A, Hanebaly, E: Perturbed integral equations in modular function spaces. Electron. J. Qual. Theory Differ. Equ. 20, 1-7 (2003). http://www.math.u-szeged.hu/ejqtde/

9. Hussain, N, Khamsi, MA: On asymptotic pointwise contractions in metric spaces. Nonlinear Anal. 71(10), 4423-4429 (2009)

10. Ishikawa, S: Fixed points by a new iteration method. Proc. Am. Math. Soc. 44, 147-150 (1974)

11. Kaminska, A: On uniform convexity of Orlicz spaces. Indag. Math. 44(1), 27-36 (1982)

12. Khan, AR: On modified Noor iterations for asymptotically nonexpansive mappings. Bull. Belg. Math. Soc. Simon Stevin $17,127-140(2010)$

13. Khamsi, MA: Nonlinear semigroups in modular function spaces. Math. Jpn. 37(2), 1-9 (1992)

14. Khamsi, MA: Fixed point theory in modular function spaces. In: Proceedings of the Workshop on Recent Advances on Metric Fixed Point Theory Held in Sevilla, September, 1995, pp. 31-35 (1995)

15. Khamsi, MA: A convexity property in Modular function spaces. Math. Jpn. 44(2), 269-279 (1996)

16. Khamsi, MA: On asymptotically nonexpansive mappings in hyperconvex metric spaces. Proc. Am. Math. Soc. 132, 365-373 (2004)

17. Khamsi, MA, Kozlowski, WM: On asymptotic pointwise contractions in modular function spaces. Nonlinear Anal. 73 , 2957-2967 (2010)

18. Khamsi, MA, Kozlowski, WM: On asymptotic pointwise nonexpansive mappings in modular function spaces. J. Math. Anal. Appl. 380(2), 697-708 (2011)

19. Khamsi, MA, Kozlowski, WM, Reich, S: Fixed point theory in modular function spaces. Nonlinear Anal. 14, 935-953 (1990)

20. Khamsi, MA, Kozlowski, WM, Shutao, C: Some geometrical properties and fixed point theorems in Orlicz spaces. J. Math. Anal. Appl. 155(2), 393-412 (1991)

21. Kirk, WA, Xu, HK: Asymptotic pointwise contractions. Nonlinear Anal. 69, 4706-4712 (2008)

22. Kozlowski, WM: Notes on modular function spaces I. Ann. Soc. Math. Pol., 1 Comment. Math. 28, 91-104 (1988)

23. Kozlowski, WM: Notes on modular function spaces II. Ann. Soc. Math. Pol., 1 Comment. Math. 28, 105-120 (1988)

24. Kozlowski, WM: Modular Function Spaces. Series of Monographs and Textbooks in Pure and Applied Mathematics, vol. 122. Dekker, New York (1988)

25. Kozlowski, WM: Fixed point iteration processes for asymptotic pointwise nonexpansive mappings in Banach spaces. J. Math. Anal. Appl. 377, 43-52 (2011)

26. Kozlowski, WM: Common fixed points for semigroups of pointwise Lipschitzian mappings in Banach spaces. Bull. Aust. Math. Soc. 84, 353-361 (2011)

27. Kozlowski, WM: On the existence of common fixed points for semigroups of nonlinear mappings in modular function spaces. Ann. Soc. Math. Pol., 1 Comment. Math. 51(1), 81-98 (2011)

28. Kozlowski, WM, Sims, B: On the convergence of iteration processes for semigroups of nonlinear mappings in Banach spaces (to appear)

29. Mann, WR: Mean value methods in iteration. Proc. Am. Math. Soc. 4, 506-510 (1953)

30. Nanjaras, B, Panyanak, B: Demiclosed principle for asymptotically nonexpansive mappings in CAT(0) spaces. Fixed Point Theory Appl. 2010, 268780 (2010)

31. Noor, MA, Xu, B: Fixed point iterations for asymptotically nonexpansive mappings in Banach spaces. J. Math. Anal. Appl. 267, 444-453 (2002)

32. Passty, GB: Construction of fixed points for asymptotically nonexpansive mappings. Proc. Am. Math. Soc. 84, 212-216 (1982)

33. Rhoades, BE: Fixed point iterations for certain nonlinear mappings. J. Math. Anal. Appl. 183, 118-120 (1994)

34. Samanta, SK: Fixed point theorems in a Banach space satisfying Opial's condition. J. Indian Math. Soc. 45, 251-258 (1981)

35. Schu, J: Iterative construction of fixed points of asymptotically nonexpansive mappings. J. Math. Anal. Appl. 158, 407-413 (1991)

36. Schu, J: Weak and strong convergence to fixed points of asymptotically nonexpansive mappings. Bull. Aust. Math. Soc. 43, 153-159 (1991)

37. Shutao, C: Geometry of Orlicz Spaces. Dissertationes Mathematicae, vol. 356 (1996)

38. Tan, $\mathrm{K}-\mathrm{K}, \mathrm{Xu}, \mathrm{H}-\mathrm{K}$ : The nonlinear ergodic theorem for asymptotically nonexpansive mappings in Banach spaces. Proc. Am. Math. Soc. 114, 399-404 (1992) 
39. Tan, $\mathrm{K}-\mathrm{K}, \mathrm{Xu}, \mathrm{H}-\mathrm{K}$ : A nonlinear ergodic theorem for asymptotically nonexpansive mappings. Bull. Aust. Math. Soc. 45, 25-36 (1992)

40. Tan, K-K, Xu, H-K: Approximating fixed points of nonexpansive mappings by the Ishikawa iteration process. J. Math. Anal. Appl. 178, 301-308 (1993)

41. Tan, K-K, Xu, H-K: Fixed point iteration processes for asymptotically nonexpansive mappings. Proc. Am. Math. Soc. $122,733-739$ (1994)

42. $\mathrm{Xu}, \mathrm{H}-\mathrm{K}$ : Existence and convergence for fixed points of asymptotically nonexpansive type. Nonlinear Anal. 16, 1139-1146 (1991)

doi:10.1186/1687-1812-2012-118

Cite this article as: Dehaish and Kozlowski: Fixed point iteration processes for asymptotic pointwise nonexpansive mapping in modular function spaces. Fixed Point Theory and Applications 2012 2012:118.

Submit your manuscript to a SpringerOpen ${ }^{\circ}$ journal and benefit from:

- Convenient online submission

- Rigorous peer review

- Immediate publication on acceptance

Open access: articles freely available online

- High visibility within the field

- Retaining the copyright to your article

Submit your next manuscript at $\boldsymbol{\nabla}$ springeropen.com 